\title{
Automated Generation of Finite-Element Meshes for Aircraft Conceptual Design
}

\author{
$\mathrm{Wu} \mathrm{Li}{ }^{1}$ and Jay Robinson ${ }^{2}$ \\ NASA Langley Research Center, Hampton, Virginia 23681, USA
}

\begin{abstract}
This paper presents a novel approach for automated generation of fully connected finiteelement meshes for all internal structural components and skins of a given wing-body geometry model, controlled by a few conceptual-level structural layout parameters. Internal structural components include spars, ribs, frames, and bulkheads. Structural layout parameters include spar/rib locations in wing chordwise/spanwise direction and frame/bulkhead locations in longitudinal direction. A simple shell thickness optimization problem with two load conditions is used to verify versatility and robustness of the automated meshing process. The automation process is implemented in ModelCenter starting from an OpenVSP geometry and ending with a NASTRAN 200 solution. One subsonic configuration and one supersonic configuration are used for numerical verification. Two different structural layouts are constructed for each configuration and five finite-element meshes of different sizes are generated for each layout. The paper includes various comparisons of solutions of 20 thickness optimization problems, as well as discussions on how the optimal solutions are affected by the stress constraint bound and the initial guess of design variables.
\end{abstract}

$\begin{array}{ll} & \\ x, y, z & =\text { coordinates of a point in a three-dimensional space } \\ \text { FEA } & =\text { finite-element analysis } \\ \text { FEM } & =\text { finite-element model } \\ \text { LE } & =\text { leading edge of wing } \\ \text { TE } & =\text { trailing edge of wing }\end{array}$

\section{Introduction}

FULL aircraft finite-element analysis (FEA) is labor-intensive with a relatively long turn-around time (months or years). Available automation approaches for FEA during aircraft conceptual design are primarily developed for individual aircraft components. Internal structural components (such as spars and ribs) are generated for one aircraft component (such as a wing) using a parametric scheme, and fully connected finite-element meshes can be automatically generated for the aircraft component (see, e.g., Refs. 1 and 2). Here, a fully connected finite-element mesh means that every two connected elements share the same nodes at the common edge. In contrast, there are other methods that can virtually connect two disconnected elements with constraints. The virtual connectivity can be established either manually or via NASTRAN ${ }^{3}$ glue operation. ${ }^{4}$ There are also efforts to automatically generate a fully connected finite-element mesh for multiple aircraft components such as fuselage and wing. RapidFEM ${ }^{5}$ represents the state-of-the-art in automated generation of fully connected finite-element meshes for multiple aircraft components. It uses a 2D sketch to define the internal structural components, generates a finite-element mesh for each aircraft component, and applies a merge algorithm to combine component meshes into one fully connected finite-element mesh; however, any automation approach based on merging or gluing component meshes faces two key technical challenges: (1) automatically connecting internal structural elements to form proper load paths and (2) avoiding triangles of poor aspect ratios when connecting different aircraft components.

This paper presents a novel approach for automated generation of a fully connected finite-element mesh for fuselage and aerodynamic surfaces of an aircraft configuration. In theory, this approach can be applied to any component-based geometry model in OpenVSP ${ }^{6}$ Hermite format, ${ }^{7}$ but the current implementation is only completed

\footnotetext{
${ }^{1}$ Senior Research Engineer, Aeronautics Systems Analysis Branch, Mail Stop 442

${ }^{2}$ Aerospace Engineer, Aeronautics Systems Analysis Branch, Mail Stop 442
}

American Institute of Aeronautics and Astronautics 
for fuselage and wing to demonstrate the feasibility of such an automation process. The key idea is to generate a geometry model for internal structural components and skins such that any two connected surfaces share a common boundary curve. Such a geometry model will be called "FEM-ready" geometry because it enables automated generation of a fully connected finite-element mesh.

The envisioned process for automation of FEA for aircraft conceptual design can be summarized as follows:

1. Specify conceptual-level layout parameters for internal structural components.

2. Generate a fully connected surface geometry model (FEM-ready geometry) for internal structural components and skins using piecewise linear approximations of the original geometry model.

3. Identify watertight compartments using the surfaces for internal structural components and skins.

4. Apply the advancing front method ${ }^{8}$ in Geompack $++^{9}$ to generate a fully connected quadrilateral mesh or HYBRID mesher in PATRAN ${ }^{10}$ to generate a fully connected finite-element mesh for the collection of watertight compartments.

5. Assign all structural elements to predefined groups.

6. Select material properties for the predefined groups using a knowledge-based material database.

7. Create aero panels for fuselage and aerodynamic surfaces using piecewise linear approximations.

8. Couple aero panels and structural elements using splines and 2D projection mappings.

9. Add concentrated masses to model other components of the airplane configuration such as landing gear, engines, and fuel tanks.

10. Define load conditions using a knowledge-based load database.

11. Generate NASTRAN decks of the inputs for NASTRAN aeroelastic analysis or optimization.

A watertight compartment is very similar to a watertight outer mold line for computational fluid dynamics surface meshing. The only difference is that a watertight compartment might contain internal surfaces (see subsection II.A). The aero-structure coupling is out of the scope of this paper. Instead, steps 7-10 are replaced with two simple load cases of applying constant pressure on the wing surface upward and downward, respectively.

The resulting automated FEA process is implemented in ModelCenter ${ }^{11}$ (see Fig. 1). The automation process (i) starts with an OpenVSP geometry model (LoadGeoFile), (ii) uses a set of conceptual-level layout parameters to generate the FEM-ready geometry with internal structural components (conceptFEM), (iii) generates a fully connected finite-element mesh (conceptFEM), (iv) specifies upward and downward constant wing pressure loads, as well as the bound for von Mises stress on all elements (SizingInputs), (v) uses the thickness of each partition surface in the FEMready geometry as a design variable, specifies the bounds for design variables, and chooses an initial guess for design variables (SizingInputs), (vi) defines material properties for 16 different predefined groups of elements (SizingInputs and materialsDB), (vii) generates the corresponding NASTRAN bulk data for thickness optimization (conceptSizing), and (viii) runs NASTRAN design optimization (conceptSizing).

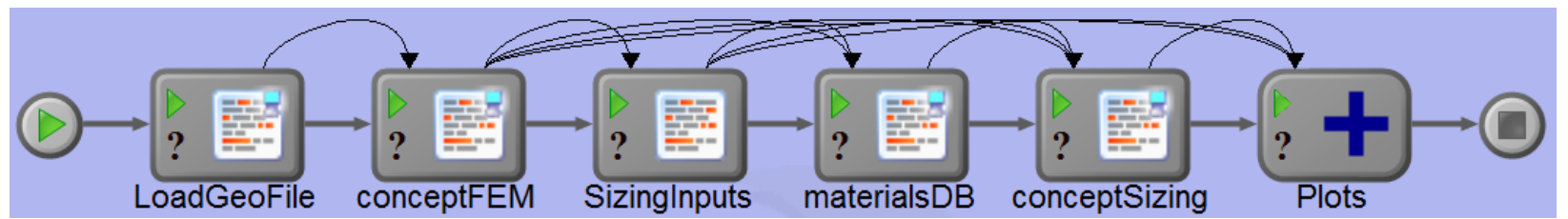

Figure 1. ModelCenter process for automated FEA.

Section II introduces the numerical methods used in the automated FEA process, including the construction of FEM-ready geometry, automated finite-element meshing, and formulation and solution of the thickness optimization problem. Detailed discussions on user input requirements to run the process are given in Section III. One supersonic configuration and one subsonic configuration are used for numerical verification of the automation process. Two different structural layouts are constructed for each configuration and five finite-element meshes of different sizes are generated for each layout. Section IV includes various comparisons of solutions of the 20 thickness optimization problems, as well as discussions on how the optimal solutions are affected by stress constraint bound and initial guess of design variables. Concluding remarks are given in Section V.

\section{Automated FEA Process}

The analysis component conceptFEM in Fig. 1 constructs FEM-ready geometry and generates a finite-element mesh using a set of conceptual-level structural layout parameters. Material assignments are performed by materialsDB in Fig. 1. All the required parameters for setting up a thickness optimization problem under two constant pressure load conditions on the wing are fed to the design component conceptSizing, which calculates optimal thickness distribution 
and optimal structural weight. However, conceptSizing could also use a given set of thickness parameters without thickness optimization and get a linear static (NASTRAN 101) solution. Tecplot ${ }^{12}$ is used to plot FEM-ready geometry and finite-element mesh. PATRAN is used to plot NASTRAN solutions.

The numerical methods behind the automated FEA process will be described in the following three subsections. Subsection A discusses the construction of FEM-ready geometry. Subsection B introduces two meshing tools that can robustly generate fully connected finite-element meshes for a FEM-ready geometry and discusses their pros and cons. The last subsection describes the bookkeeping process of converting all generated data for thickness optimization into bulk data format for NASTRAN design optimization runs.

\section{A. Construction of FEM-Ready Geometry}

The existing automation processes ${ }^{4,5}$ for FEA of multiple aircraft components are based on merging/gluing finiteelement meshes of aircraft components after construction of finite-element meshes for every component independently. Here is a novel approach of separating construction of internal structural components and generation of finite-element meshes. The key idea is to construct a fully connected geometry model that contains all internal components. Such a geometry model is referred to as FEM-ready geometry. A FEM-ready geometry model not only contains all internal structural components, but also satisfies the condition that any two connected surfaces share the same boundary curve.

The main motivation for introducing FEM-ready geometry is that several meshing tools can be used to generate a fully connected finite-element mesh of a FEM-ready geometry without any further manual preprocessing of the geometry (see the next subsection). The separation of internal structural component construction and meshing avoids the merge of disconnected structural meshes. Instead, the construction of FEM-ready geometry only needs to deal with the connectivity of various structural components and generates the desired layout topology, while leaving the meshing problem to professionally/commercially developed meshing tools. This strategy will ensure the use of the state-of-the-art meshing technology in the automated FEA process.

A notional, $100 \mathrm{ft}$ long, low-boom supersonic flight demonstrator concept ${ }^{13}$ in OpenVSP Hermite format ${ }^{7}$ (shown in Fig. 2) is used to illustrate the numerical method for construction of a FEM-ready geometry. Each aircraft component in Hermite format is represented by a rectangular grid of points $\{(x[\mathrm{i}][\mathrm{j}], y[\mathrm{i}][\mathrm{j}], z[\mathrm{i}][\mathrm{j}]): 1 \leq \mathrm{i} \leq \mathrm{m}, 1 \leq \mathrm{j} \leq$ $\mathrm{n}\}$.

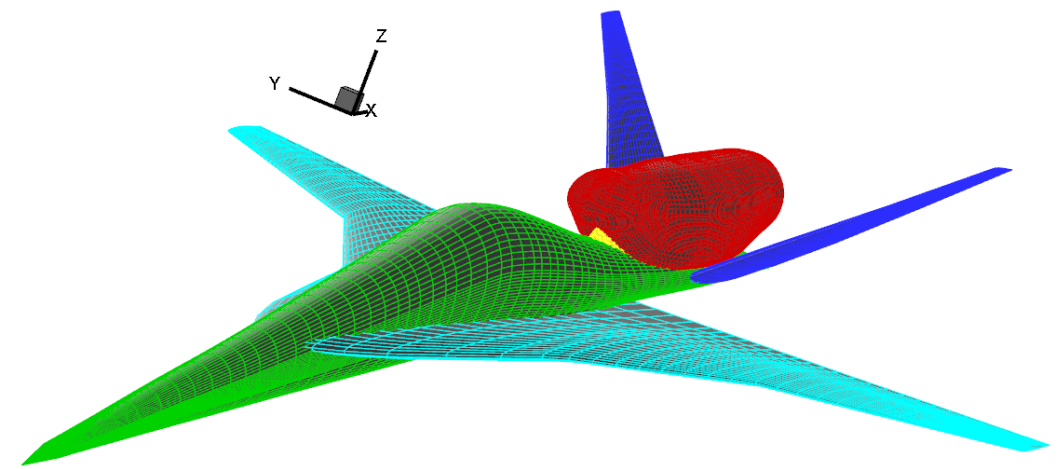

Figure 2. OpenVSP geometry model in Hermite format.

Infeasible layout parameters are detected automatically, with a visual display to show why the input parameters are infeasible. Most infeasible cases are due to spars lying outside of the wing planform and can be easily corrected once the spar locations and wing planform are displayed (see Fig. 3).

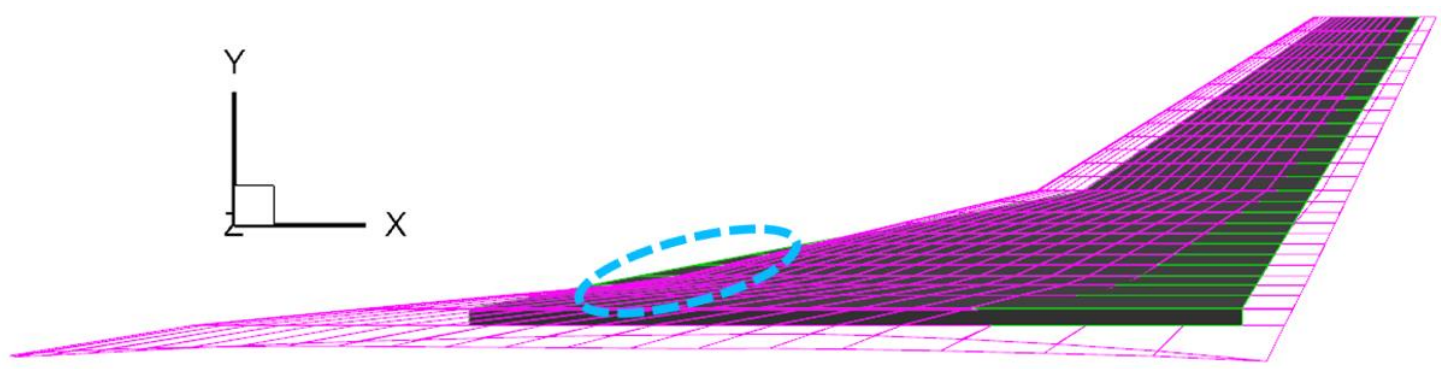

Figure 3. Front spar outside wing planform.

American Institute of Aeronautics and Astronautics 
If the layout parameters are feasible, all internal structural components are defined by using piecewise linear approximations of the original geometry model. For example, a typical spar surface outside the fuselage is bounded above by the upper wing skin surfaces, bounded below by the lower wing skin surfaces, and bounded on two sides by rib surfaces. After construction of fully connected internal structural components, new surfaces for the outer mold line of the fuselage and wing are generated (using linear approximations) so that all surfaces are fully connected for meshing. That is, if two surfaces are connected, then they share a common boundary curve. Figure 4 shows fully connected surfaces for internal structural components and skins (with some skins hidden to expose the internal structural components).

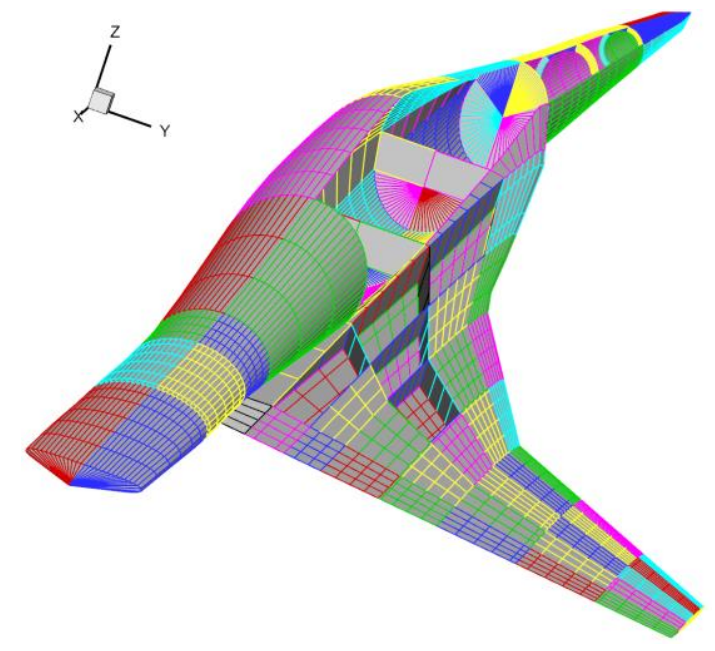

Figure 4. Fully connected surfaces (FEM-ready geometry) for internal structural components and skins.

The [discrete] geometry model in Fig. 4 can be considered as a fully connected finite-element mesh with triangles and quadrilaterals. But, in general, it cannot be used for FEA because poor aspect ratios of elements and their irregular sizes might lead to faulty FEA results.

One could also view the FEM-ready geometry as a [continuous] geometry model defined by bilinear B-splines. ${ }^{14}$ Then various finite-element meshes for the underlying bilinear B-spline geometry can be easily generated using PATRAN's meshers without any further preprocessing of the geometry model. See the next subsection for details.

Another option to automatically generate a fully connected quadrilateral mesh for the FEM-ready geometry is to use the advancing front method in the meshing software Geompack++. For quadrilateral surface meshing, Geompack++ requires a decomposition of the input geometry into watertight compartments with proper orientations of surface normal. A pattern recognition algorithm is developed to rearrange all surfaces into watertight compartments.

A watertight compartment is very similar to a watertight outer mold line. The only difference is that a watertight compartment might contain internal surfaces. Figure 5 shows the most complicated watertight compartment for the notional low-boom demonstrator concept with four spars and cutouts above them inside the fuselage. Four fuselage skin surfaces are hidden in Fig. 5 to expose the internal surfaces, which include ring surfaces (for a frame) at the trailing edge of the fuselage/wing intersection and surfaces for four spars inside the fuselage.
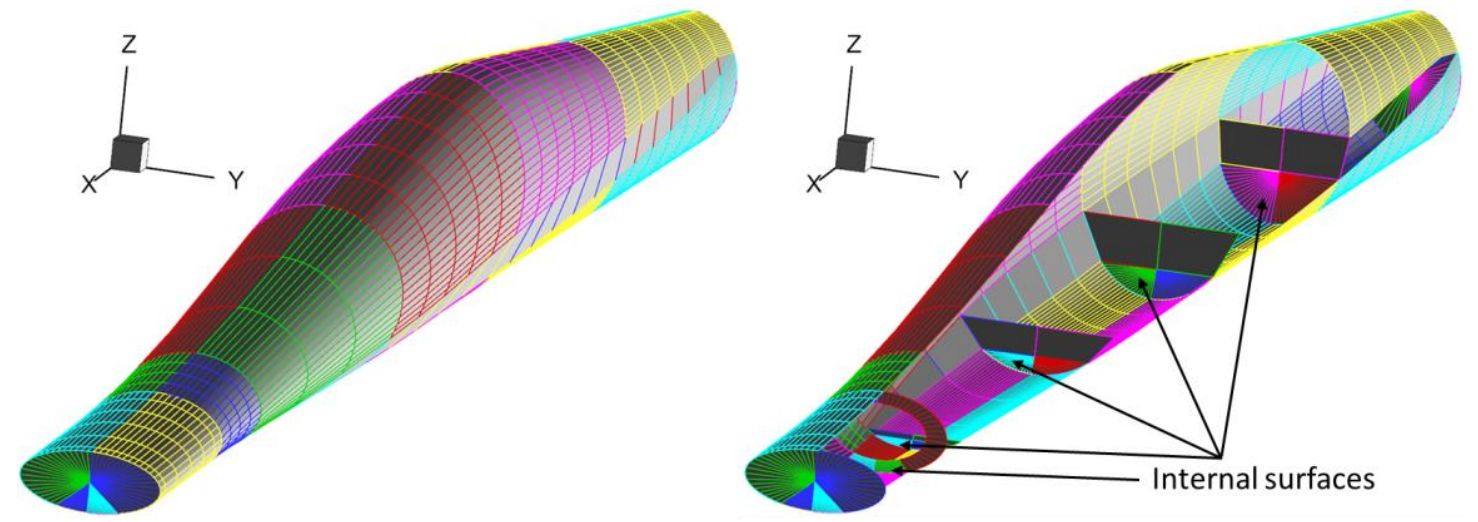

Figure 5. A watertight compartment [with some fuselage skin surfaces hidden].

4

American Institute of Aeronautics and Astronautics 


\section{B. Automated Finite-Element Meshing}

The current automation process uses the HYBRID mesher in PATRAN and the advancing front method in Geompack++ to generate fully connected finite-element meshes for any FEM-ready geometry. HYBRID can generate finite-element meshes of various sizes by using a global length control parameter, but the generated mesh usually contains a few triangles. The advancing front method in Geompack++ can always generate a fully connected quadrilateral mesh, but it is not easy to control the mesh size and quality. Both meshing tools might fail to generate a mesh if the control parameters are set for a mesh with extremely high resolution.

The implemented PATRAN meshing process is controlled by PCL commands in a session file. The surfaces in a FEM-ready geometry are organized by 16 predefined groups (see Section III for details). Each group of surfaces is exported to one IGES ${ }^{15}$ file with multiple bilinear B-spline surfaces. Only surfaces in the half space $y \geq 0$ are exported. With the same set of control parameters for PATRAN meshing and uniform grid point distributions on surface edges, all surface meshes will have the same grid points on their common boundary curves. Using the "equivalence" function call within PATRAN, all redundant nodes generated by meshing each surface independently are eliminated, and the resulting mesh in the half space $y \geq 0$ is fully connected (because the imported FEM-ready geometry is fully connected).

To avoid any confusion from internal labeling by PATRAN, the finite-element meshes for different predefined groups are exported separately and the exported meshes are retagged to maintain the original FEM-ready geometry data structure. All shell elements for each surface in the FEM-ready geometry share the same surface tag and all shell elements for all surfaces in one predefined group share the same material tag. This allows automation of material property assignments and design variable definitions for all shell elements. In addition, it is very convenient to use one thickness design variable for all elements with the same surface tag. Such a definition of design variable is meshindependent, which allows a meaningful study on mesh sensitivity for the thickness optimization problem later.

FEM-ready geometry is also exported in the $3 \mathrm{D}$ region format ${ }^{16}$ required by Geompack++, after all watertight compartments are identified and all surfaces are represented by bilinear B-splines. Then, Geompack++ reads the geometry in the 3D region format and uses a few control parameters to generate a quadrilateral mesh for the internal structural components and skins. The generated quadrilateral mesh will maintain the original FEM-ready geometry data structure. The post-processing of the generated mesh only involves removal of quadrilaterals in the half space $y$ $\leq 0$.

Both meshing tools are very robust in generating a finite-element mesh for any given FEM-ready geometry. Figure 6 shows a finite-element mesh of 9,567 nodes, 10,372 quadrilaterals, and 299 triangles generated by HYBRID mesher in PATRAN, while Fig. 7 shows a quadrilateral mesh of 14,947 nodes and 15,917 quadrilaterals generated by the advancing front method in Geompack++. In each figure, the shell elements are grouped by different surface tags and the plot on the right shows the internal structural elements.

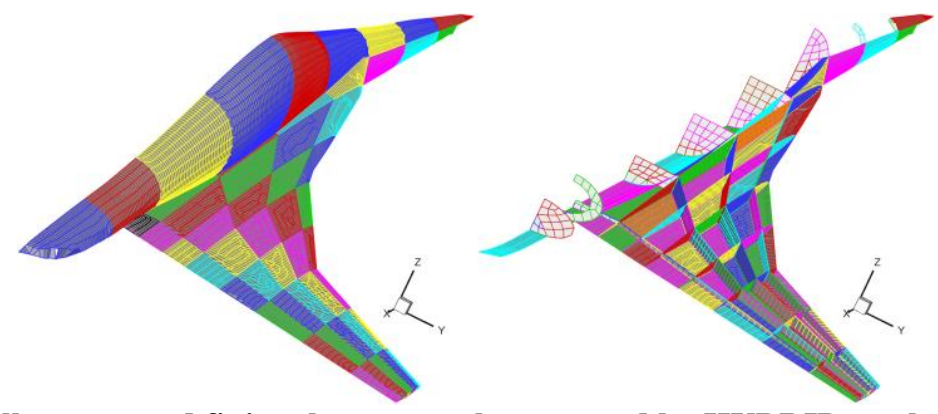

Figure 6. A fully connected finite-element mesh generated by HYBRID mesher in PATRAN.

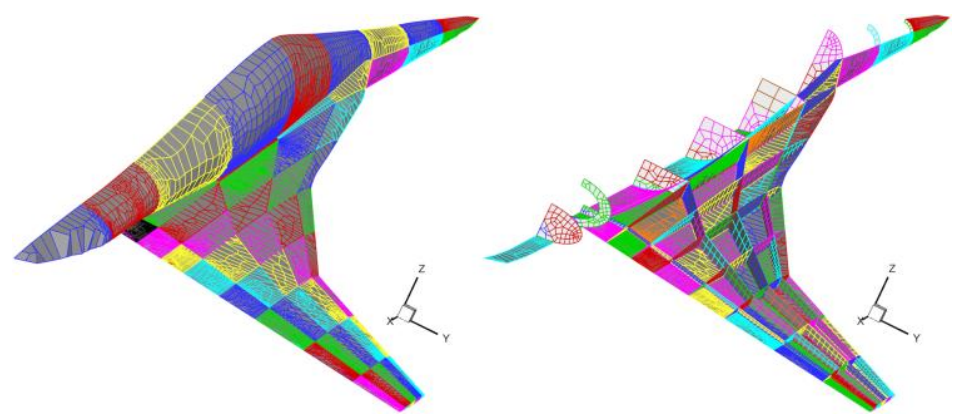

Figure 7. A fully connected quadrilateral mesh generated by the advancing front method in Geompack++.

American Institute of Aeronautics and Astronautics 


\section{Formulation and Solution of Thickness Optimization Problem}

The thickness optimization problem uses two constant pressure loads on the lower wing surface. NASTRAN bulk data entity PLOAD defines the constant pressure on a wing surface element. The values of two constant pressure loads are chosen to mimic the $3 \mathrm{G}$ pull up and $-1 \mathrm{G}$ push over load cases for structural sizing. The total wing load in the upward direction is $3 \mathrm{X}$ of the cruise weight and the total wing load in the downward direction is $1 \mathrm{X}$ of the cruise weight. All nodes on the symmetry plane are fixed. All elements for each surface in the FEM-ready geometry share the same [NASTRAN] PSHELL entity and the thickness parameter of every PSHELL entity is a design variable. All elements have the same material property of a generic aluminum alloy. A bound for the absolute value of von Mises stress for all elements is specified for the stress constraint and the objective of the thickness optimization problem is to minimize the structural weight.

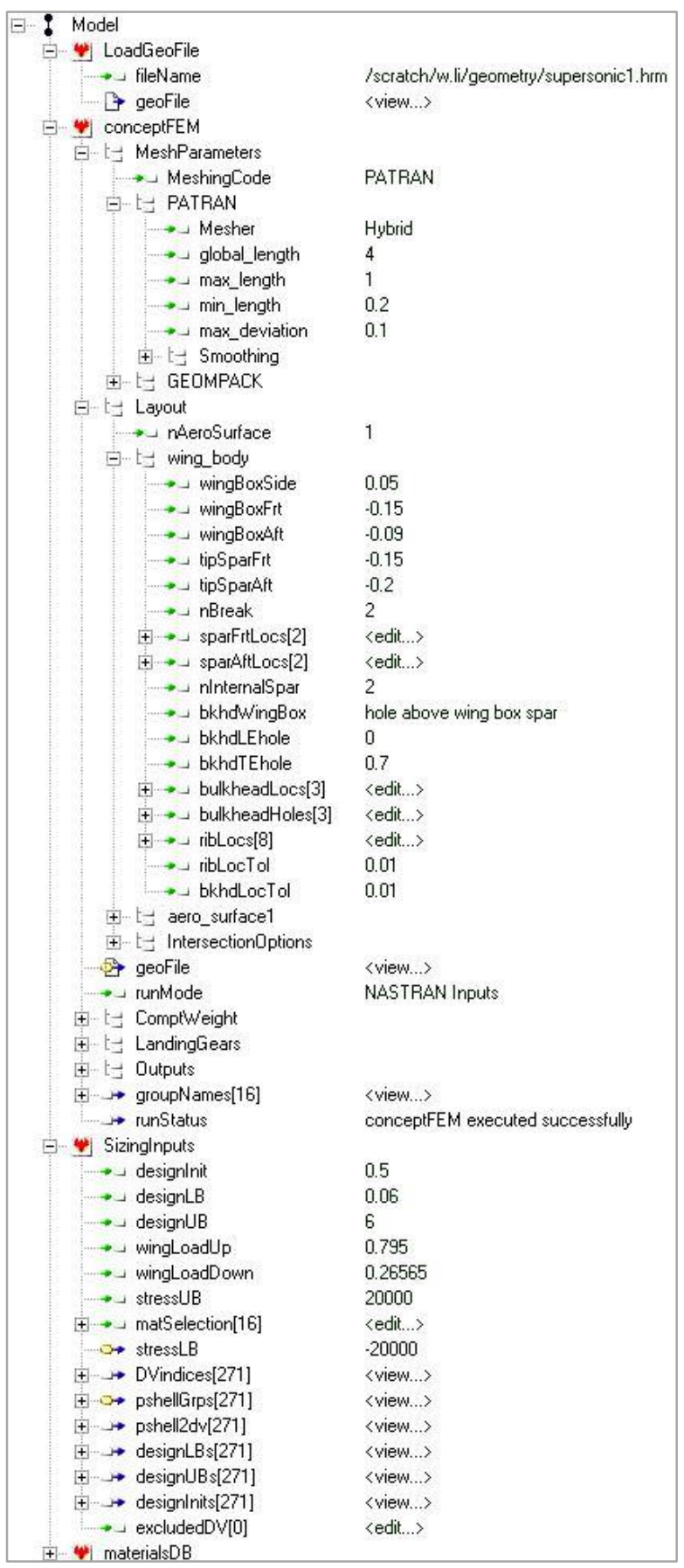

Figure 8. Key input parameters for automated FEA.
Once the initial guess and bounds for the design variables are given, a bulk data file for NASTRAN 200 solution of the corresponding thickness optimization problem (using linear static analysis) is automatically generated and NASTRAN is used to compute the optimal thickness distribution.

The process can also be used to automatically generate a linear static (NASTRAN 101) solution for a given set of thickness parameters.

\section{User Input Requirements for Automated FEA}

The automated FEA process is developed with emphasis on the following three key features: (i) minimum user input requirements, (ii) versatility (i.e., applicable to a variety of configurations), and (iii) robustness (i.e., never fail without any known limitation). This section provides an example of what info are required to run the automated FEA.

Figure 8 lists all input parameters (indicated by the green arrows) that are required to construct a generic fuselage-wing structural layout, generate a finite-element mesh, set up the thickness optimization problem, and get a NATRAN 200 solution. The hidden input parameters (such as mesh smoothing parameters for PATRAN mesher and meshing parameters for Geompack++) are not sensitive to configurations and users can use the default values.

Hermite format ${ }^{7}$ for OpenVSP geometry model (see Fig. 2) is used as the geometry input. The main reason is that a reasonably discretized geometry model is sufficient for conceptual-level structural analysis with quadrilateral and triangular elements. It also makes the construction of FEM-ready geometry more efficient and robust. The first analysis component in ModelCenter loads the specified Hermite geometry file for analysis (see Fig. 8).

The second analysis component conceptFEM in ModelCenter constructs the FEM-ready geometry and generates a fully connected finite-element mesh (using external meshing tools).

Fuselage and wing geometry components are identified by the name tags in Hermite file. The most time consuming part of the set-up process is to define the conceptual-level internal structural layout parameters for conceptFEM: wing box locations, front spar locations, rear spar locations, number of break points (or number of 
piecewise line segments) for spars, number of spars between front and rear spars, rib locations, frame/bulkhead locations, and whether there are holes above or below wing box spars inside fuselage. All $z$ coordinates for internal structural components are calculated from the corresponding $(x, y)$ locations.

A frame is defined as a bulkhead with a hole in the middle. Each parameter bulkheadLocs[i] specifies the actual $x$ location for a frame or bulkhead. If the parameter bulkheadHoles[i] is between 0 and 1 , the corresponding structural component at bulkheadLocs[i] is a frame and the ratio of cutout hole area to the cross-section area equals to bulkheadHoles[i] (which must be less than 1). Otherwise, the corresponding structural component at bulkheadLocs[i] is actually a solid wall.

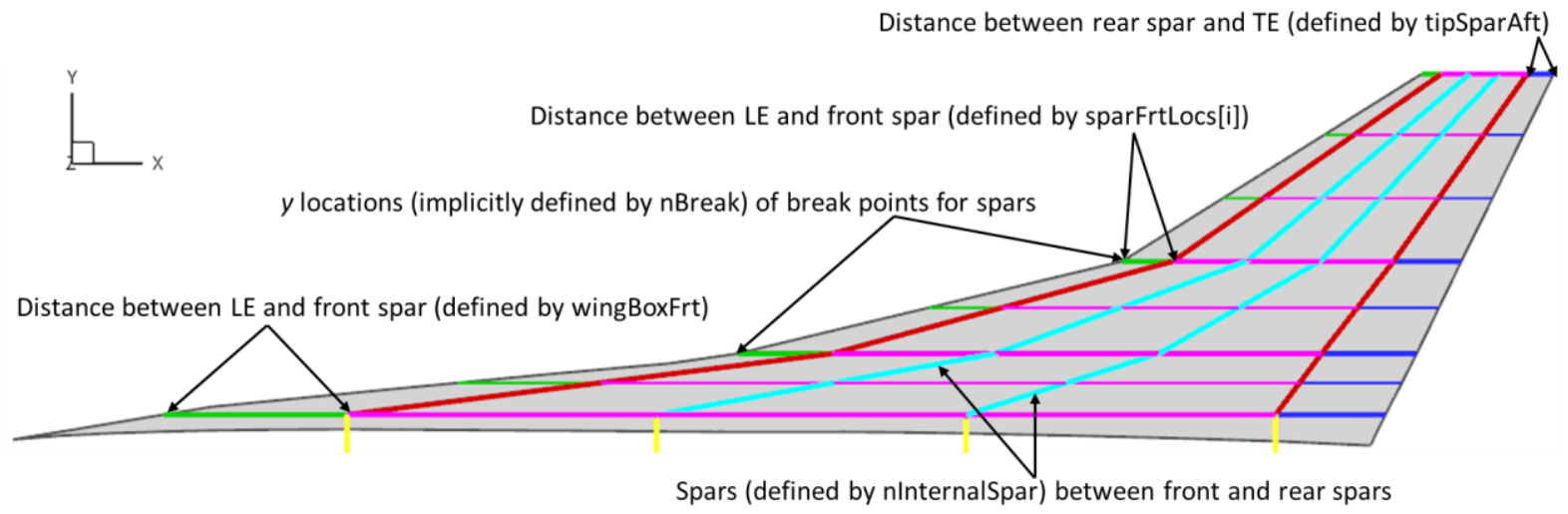

Figure 9. Illustration of some layout parameters.

Side location of wing box is defined by its distance (wingBoxSide) from the intersection of fuselage and wing. If wingBoxSide $=0$, then the side of wing box in the half space $y \geq 0$ is on the intersection curve. Front location of wing box can be specified by the absolute distance (wingBoxFrt > 0) from LE or the distance (wingBoxFrt < 0) from LE in terms of percentage of airfoil chord for side edge of wing box (see Fig. 9).

Other $x$ locations for spars are defined similarly by either the absolute distance or the distance in terms of chord length from a default reference location. All $y$ locations (except wingBoxSide) are actual $y$ coordinates for internal components.

A useful parameter for supersonic configurations is nBreak, which is the number of break points for spars. The underlying analysis code will calculate the angle between two consecutive segments of LE or TE; then find nBreak $y$ locations (see Fig. 9) where the calculated angles are smaller than the rest. The corresponding $x$ locations for front and rear spars at the selected $y$ locations for break points are defined by sparFrtLocs (see Fig. 9) and sparAftLocs. The front and rear spar locations at wing tip are defined by tipSparFrt and tipSparAft (see Fig. 9), respectively. Of course, it is relatively easy to change the user interface to allow user-specified break points for front and rear spars. Equally spaced spars between front and rear spars can be added to the layout by using input parameter nInternalSpar (under conceptFEM $\rightarrow$ Layout $\rightarrow$ wing_body in Fig. 8). For example, two spars between front and rear spars are added to the layout in Fig. 9 because nInternalSpar is 2.

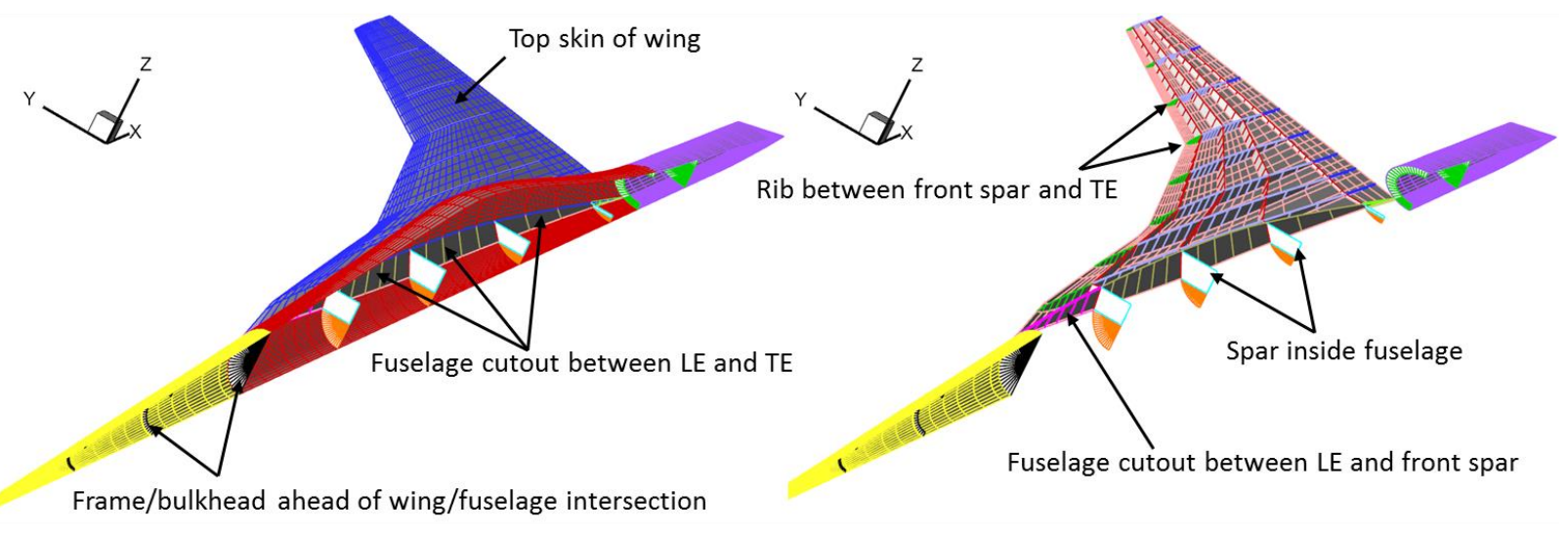

Figure 10. Illustration of some predefined groups.

American Institute of Aeronautics and Astronautics 
The current implementation arranges all surfaces in the FEM-ready geometry into 16 predefined groups (see Fig. 10): (1) top skin of wing, (2) bottom skin of wing, (3) spar inside wing, (4) spar inside fuselage, (5) rib between LE and front spar, (6) rib between rear spar and TE, (7) rib between front and rear spars, (8) fuselage skin ahead of wing/fuselage intersection, (9) fuselage skin behind wing/fuselage intersection, (10) fuselage skin along wing/fuselage intersection, (11) fuselage cutout (by wing intersection) between LE and front spar, (12) fuselage cutout (by wing intersection) between rear spar and TE, (13) fuselage cutout (by wing intersection) between front and rear spars, (14) frame/bulkhead ahead of wing/fuselage intersection, (15) frame/bulkhead behind wing/fuselage intersection, and (16) frame/bulkhead along wing/fuselage intersection. These groups allow easy assignments of material properties to all elements by specifying matSelection parameters under SizingInputs in Fig. 8. Of course, these groups can be easily redefined if more desirable grouping is needed.

The 16 groups listed above are further partitioned by intersection curves between different groups. After the partition, any two connected surfaces share a common boundary curve. Each (color-coded) surface in the FEM-ready geometry partition (see Figs. 4, 6, and 7) has one thickness design variable for thickness optimization. For example, the mesh in Fig. 6 or Fig. 7 has 195 design variables for thickness optimization (i.e., there are 195 surfaces in the half space $y \geq 0$ to define the FEM-ready geometry in Fig. 4).

This approach avoids the set-up burden of defining design variables for thickness optimization, which could be quite tedious and time consuming if the interface is not done properly.

The thickness optimization problem is to minimize the structural weight under a stress constraint. The stress constraint forces the absolute value of von Mises stress on each element to be less than or equal to a given bound for two constant pressure load conditions on the wing. To set up this simple thickness optimization problem, only six input parameters (under SizingInputs in Fig. 8) are required: the bound for von Mises stress (stressUB), the minimum gauge (designLB) for all shell elements, an optional upper bound (designUB) for thickness design variables, an initial guess (designInit) for all thickness design variables, a constant upward pressure load on the wing (wingLoadUp), and a constant downward pressure load on the wing (wingLoadDown).

The most time consuming part for set up of user input parameters is to find the appropriate parameters to define the internal structural components. For any wing-body configuration, the thickness optimization problem can be set up to run in minutes (instead of hours or days).

\section{Numerical Verification}

Two configurations are used to demonstrate versatility and robustness of the automated FEA process. One is the notional low-boom supersonic flight demonstrator shown in Fig. 2. Another is an OpenVSP model of the subsonic business jet G450 (see Fig. 11). The supersonic configuration has a cruise weight of 17,500 lbs, which corresponds to the constant upward pressure load of 0.26565 psi on the wing. The estimated fuselage and wing weights using FLOPS ${ }^{17}$ are 1,595 lbs and 1,660 lbs, respectively. The subsonic configuration has a cruise weight of 70,000 lbs, which corresponds to the constant upward pressure load of $0.7505 \mathrm{psi}$ on the wing. The estimated fuselage and wing weights of G450 using FLOPS are 5,883 lbs and 5,158 lbs, respectively.

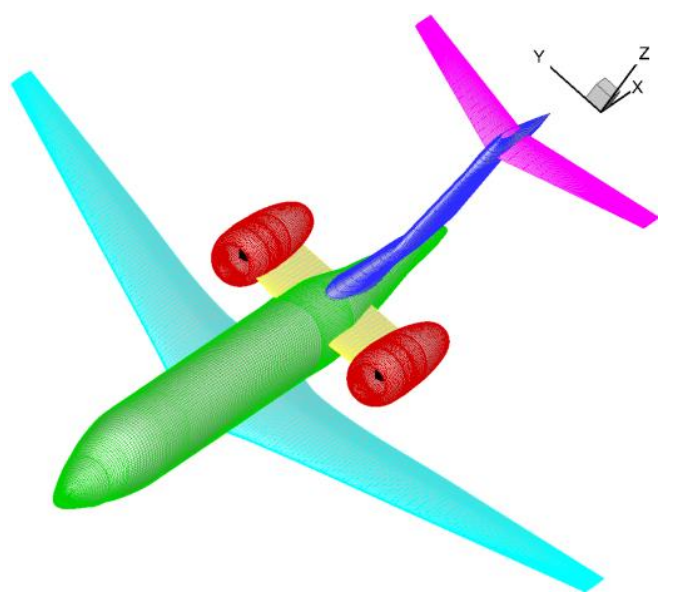

Figure 11. OpenVSP model of a subsonic business jet.

Two structural layouts with 5 and 6 spars are constructed for the supersonic configuration and two structural layouts with 3 and 4 spars are constructed for the subsonic configuration. The structural layout with 4 spars for the

American Institute of Aeronautics and Astronautics 
subsonic configuration is based on a sketch of structural layout of G450. ${ }^{18}$ The resulting finite-element meshes for these four structural layouts are shown in Figs. 12 and 13 (with all top skins of the wing hidden). These meshes are generated by HYBRID mesher in PATRAN and each mesh has approximately 17,000 elements.

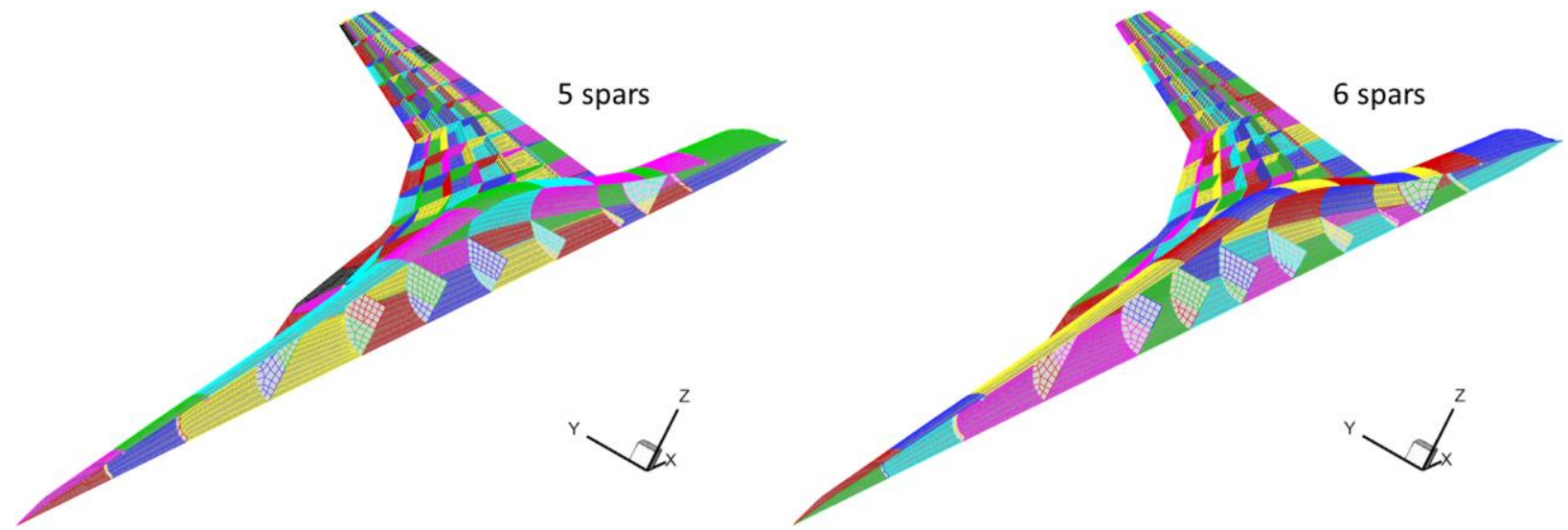

Figure 12. Finite-element meshes for the supersonic configuration

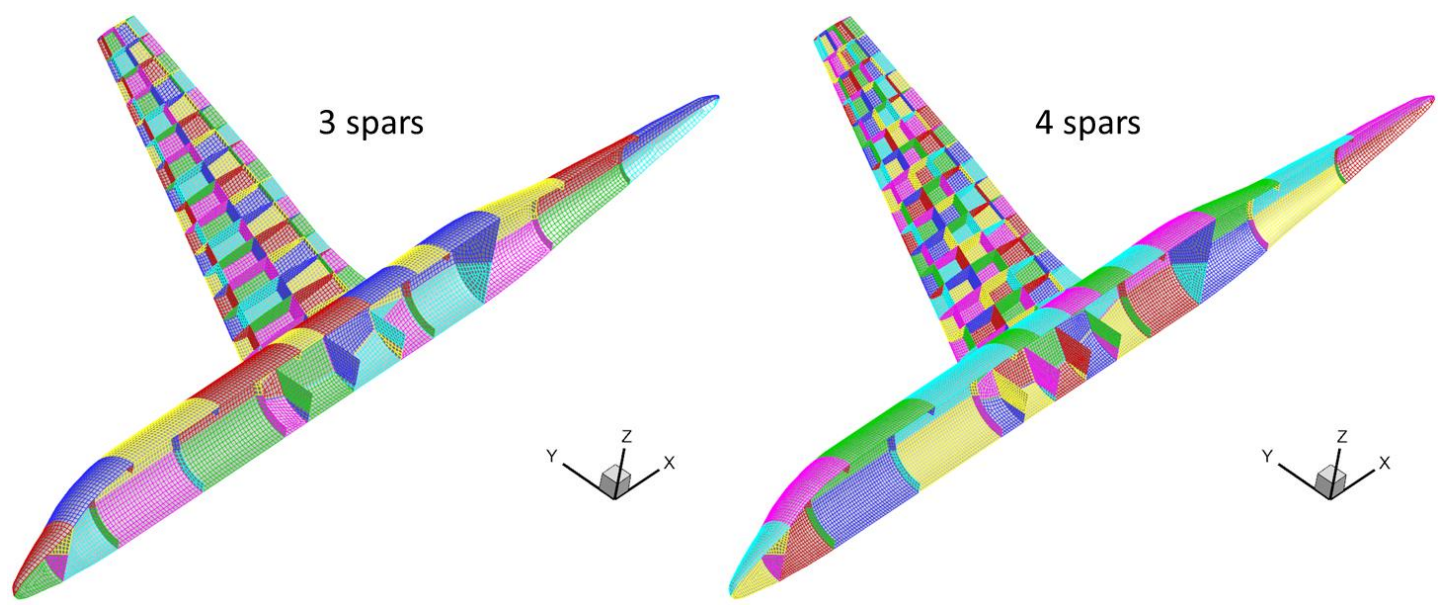

Figure 13. Finite-element meshes for the subsonic configuration

Note that it only requires changing one layout parameter nInternalSpar in Fig. 8 to get layouts with different numbers of spars. Multiple runs of design of experiments (DOE) are performed to see how the optimal structural weights are affected by the initial guess (designInit) and von Mises bound (stressUB). Figure 14 shows the DOE results for the supersonic configuration with 5 spars and the subsonic configuration with 4 spars. Hard convergence is achieved for all NASTRAN 200 runs. The initial guess has a marginal effect on the optimal structural weight. The maximum relative error for the optimal structural weight is approximately $4 \%$.
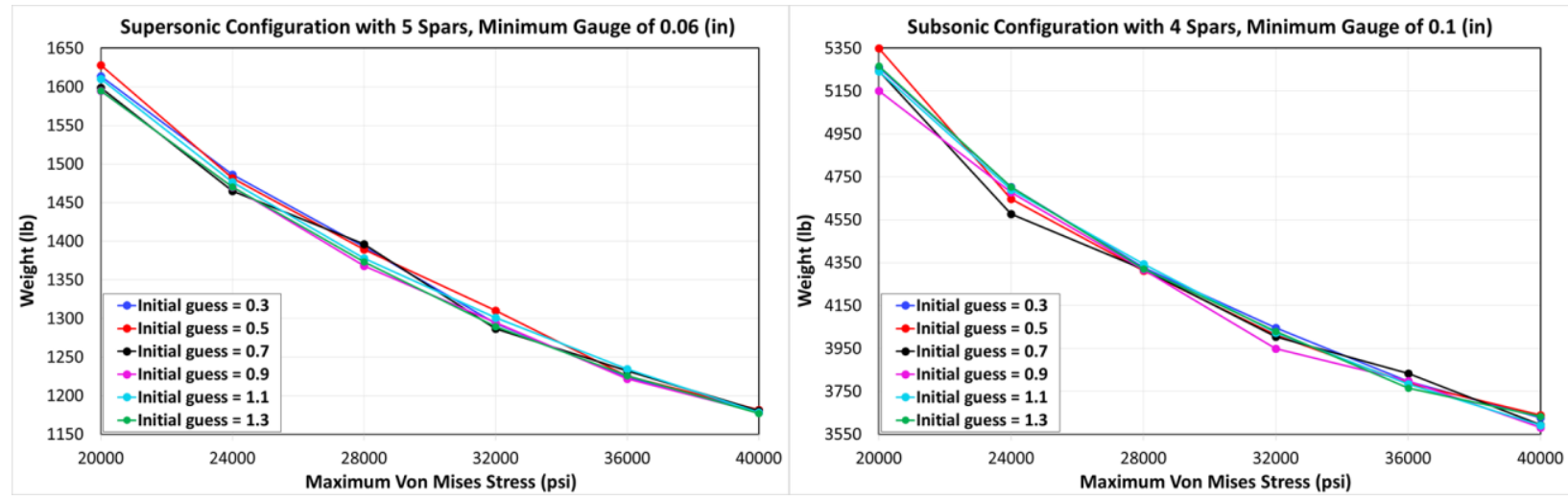

Figure 14. Sensitivity of optimal structural weight with respect to initial guess and stress bound.

American Institute of Aeronautics and Astronautics 


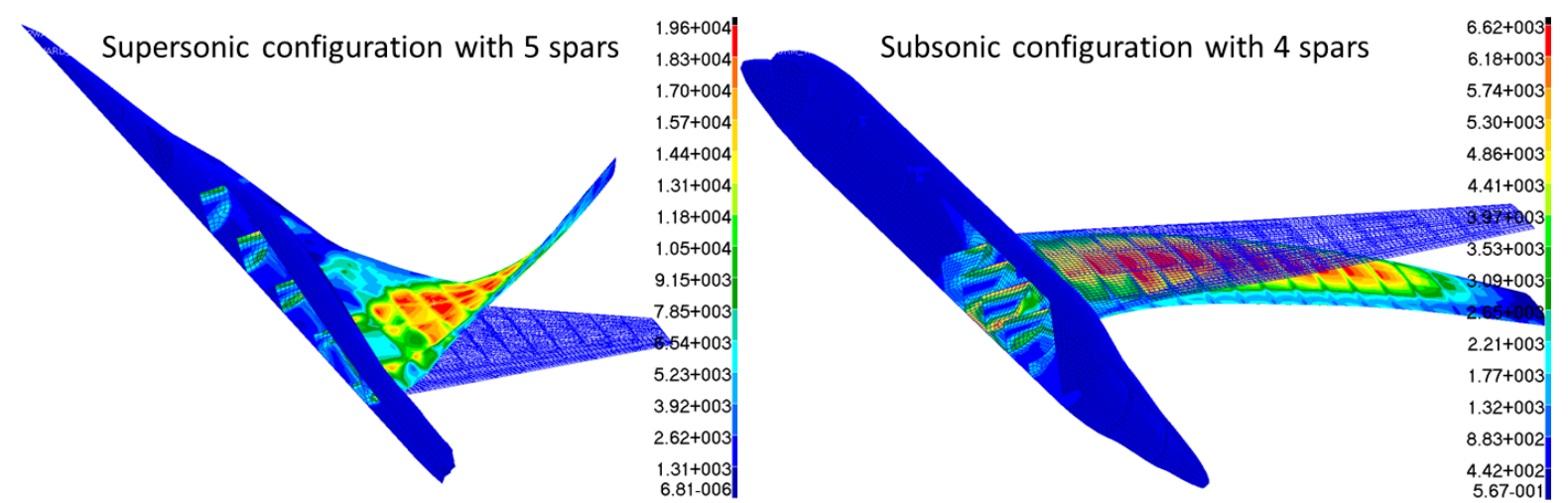

Figure 15. Von Mises stress contours and deflections of optimal structural layouts.

For von Mises bound of 20,000 psi, the stress contours for the upward pressure load on the supersonic configuration and for the downward pressure load on the subsonic configuration are shown in Fig. 15.

The total structural weights of the half models are approximately 1,650 lbs and 5,600 lbs for the supersonic and subsonic configurations, respectively, under von Mises stress bound of 20,000 psi. These values closely match the FLOPS weight estimates for these two configurations. Therefore, the von Mises stress bound of 20,000 psi is chosen for the following mesh sensitivity study.

For each layout, four meshes generated by PATRAN and one mesh generated by Geompack++ are used to study the sensitivity of the optimal structural weight with respect to the finite-element mesh used in analysis. The number of design variables for each layout ranges from 305 to 378. The results are summarized in Fig. 16.

The optimal structural weight generally increases as the PATRAN mesh size increases. The maximum deviation is approximately $11 \%$ to $16 \%$ of the optimal weight of the finest structural mesh. The relative error between the optimal weights of the two finest meshes is only $1.5 \%$ to $5.7 \%$. There are noticeable differences in optimal solutions between PATRAN and Geompack++ meshes of similar sizes.
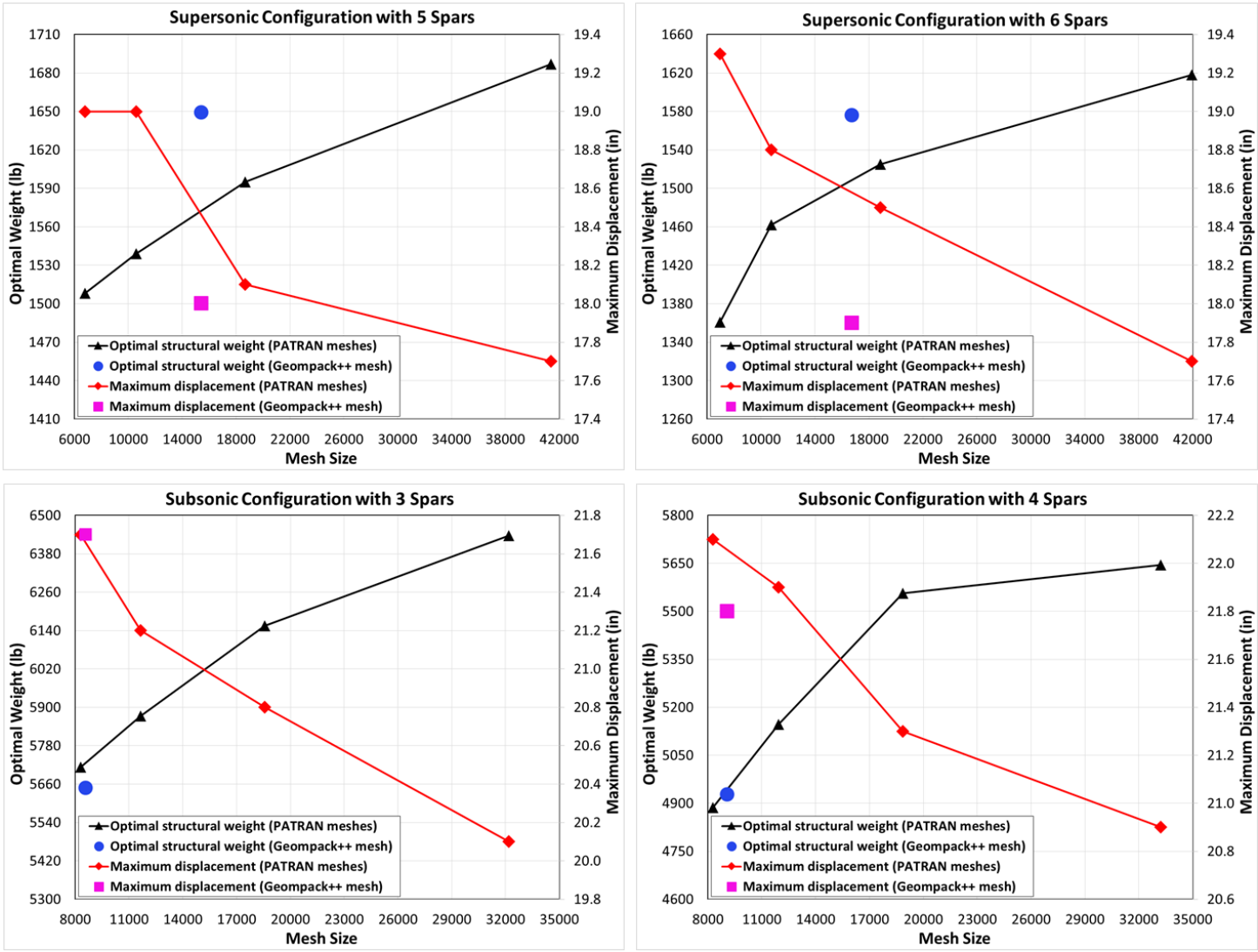

Figure 16. Sensitivity of optimal structural weight and maximum displacement to mesh size.

American Institute of Aeronautics and Astronautics 
To gain further insight on the mesh sensitivity, the optimal thickness distributions and stress contours are plotted for the coarsest and finest PATRAN meshes of the supersonic configuration with 5 spars. See Fig. 17 for the two meshes. Qualitatively, the two optimal thickness distributions are of similar characteristics in terms of where the structural elements need to be thickened to satisfy the stress constraint (see Figs. 18 and 19). Moreover, the stress contours have similar patterns: the stress contour plot for the coarsest mesh resembles a smeared version of that for the finest mesh (see Fig. 20).

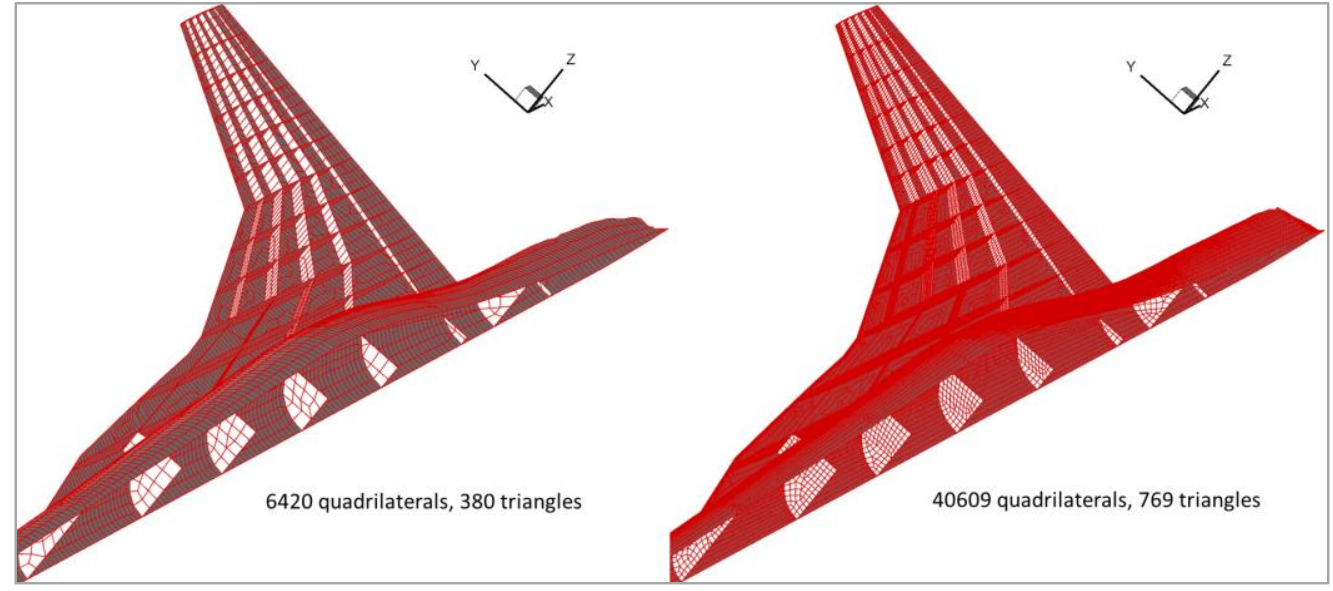

Figure 17. Coarsest and finest PATRAN meshes for supersonic configuration with 5 spars.
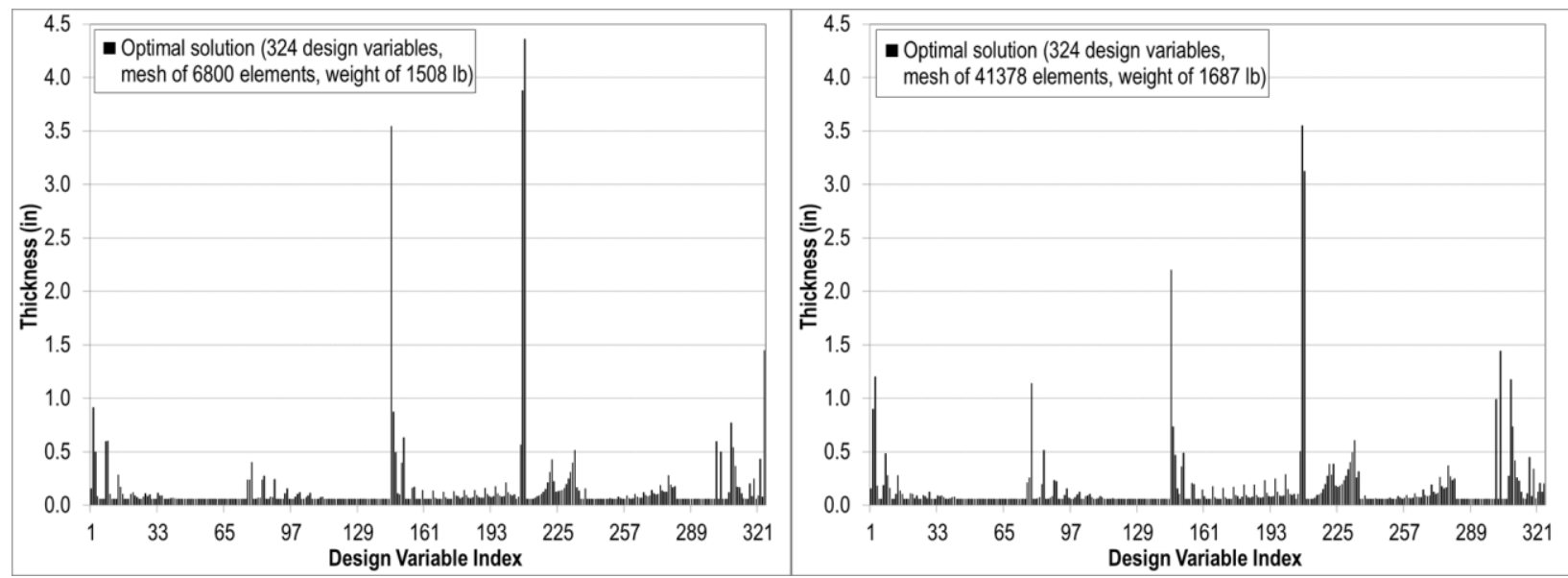

Figure 18. Optimal thickness distributions for coarsest and finest PATRAN meshes.

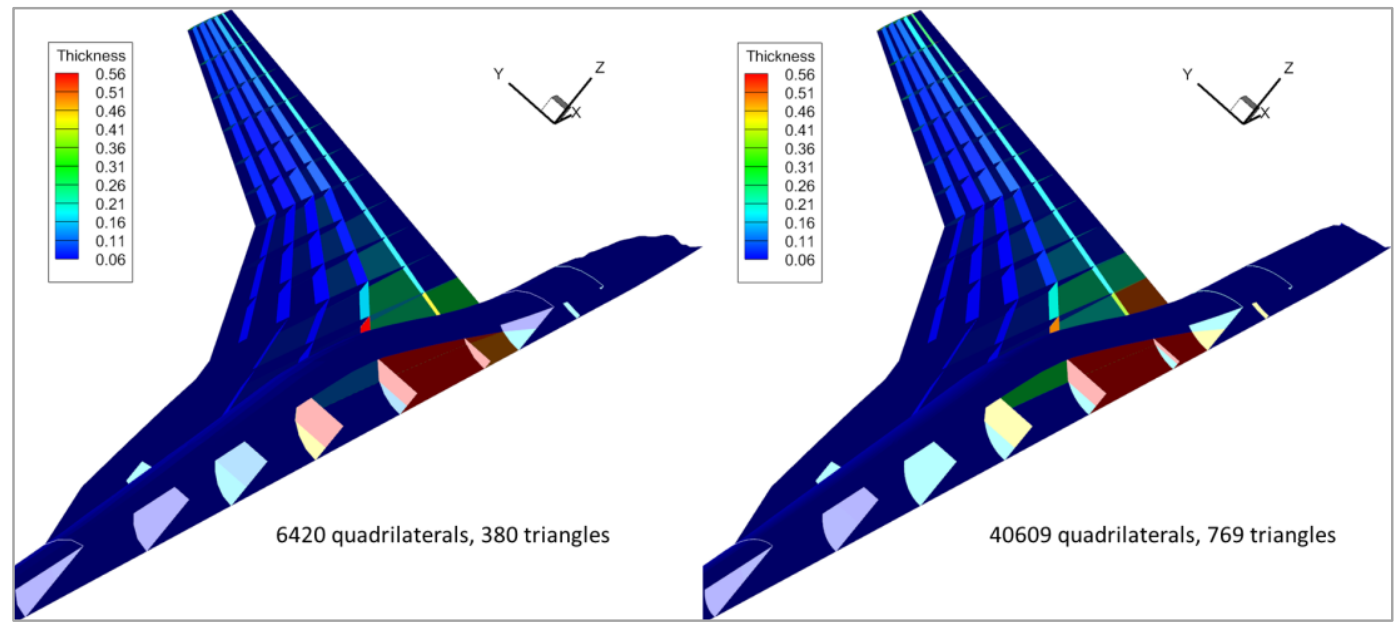

Figure 19. Optimal thickness distribution contours for coarsest and finest PATRAN meshes.

American Institute of Aeronautics and Astronautics 


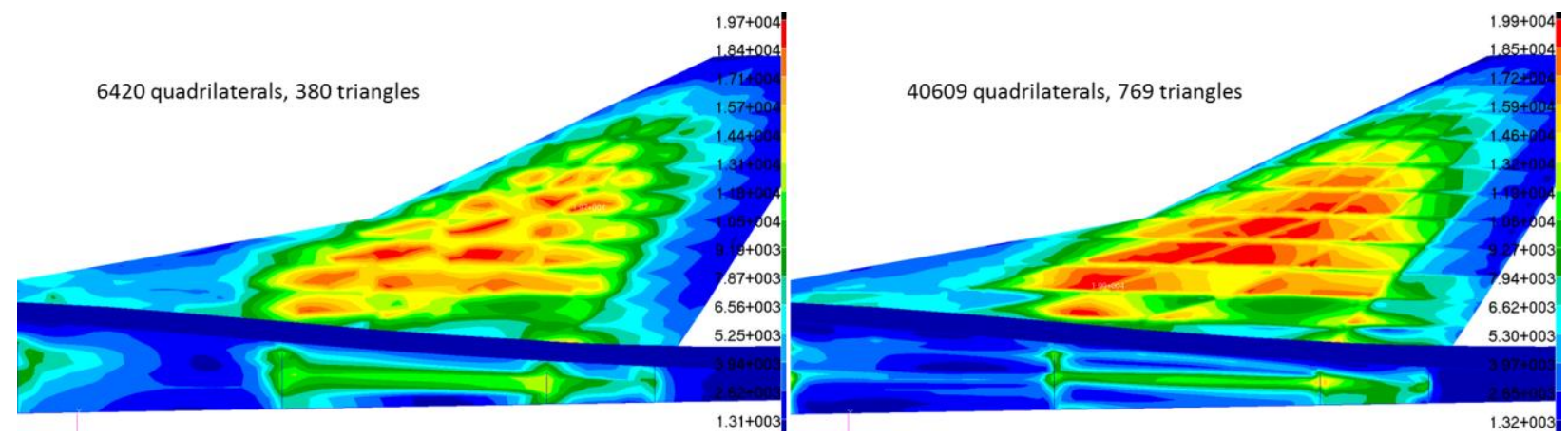

Figure 20. Von Mises stress contours for coarsest and finest PATRAN meshes.

The maximum displacement for a given thickness distribution is relatively insensitive to the five meshes used in this paper. For the supersonic configuration with 5 spars and the corresponding optimum thickness distribution generated using a PATRAN mesh of approximately 18,000 elements, the relative differences between the maximum displacements for these five meshes are less than 1.1\% (see Fig. 21). In contrast, the relative differences between the maximum von Mises stresses for PATRAN meshes are up to 15\%. The locations of the maximum von Mises stresses are different, but they are in the same vicinity inboard of the wing. The relative difference between the maximum von Mises stresses for Geompack++ mesh and any PATRAN mesh is more than $22 \%$. For the same thickness distribution, the differences between structural weights for the five meshes are less than $0.5 \%$, which are determined by the area differences of these meshes. The variability in the optimal structural weights for different meshes (shown in Fig. 16) is mainly due to the variability in the stress distributions, which the NASTRAN optimizer uses to generate the optimum thickness distribution.

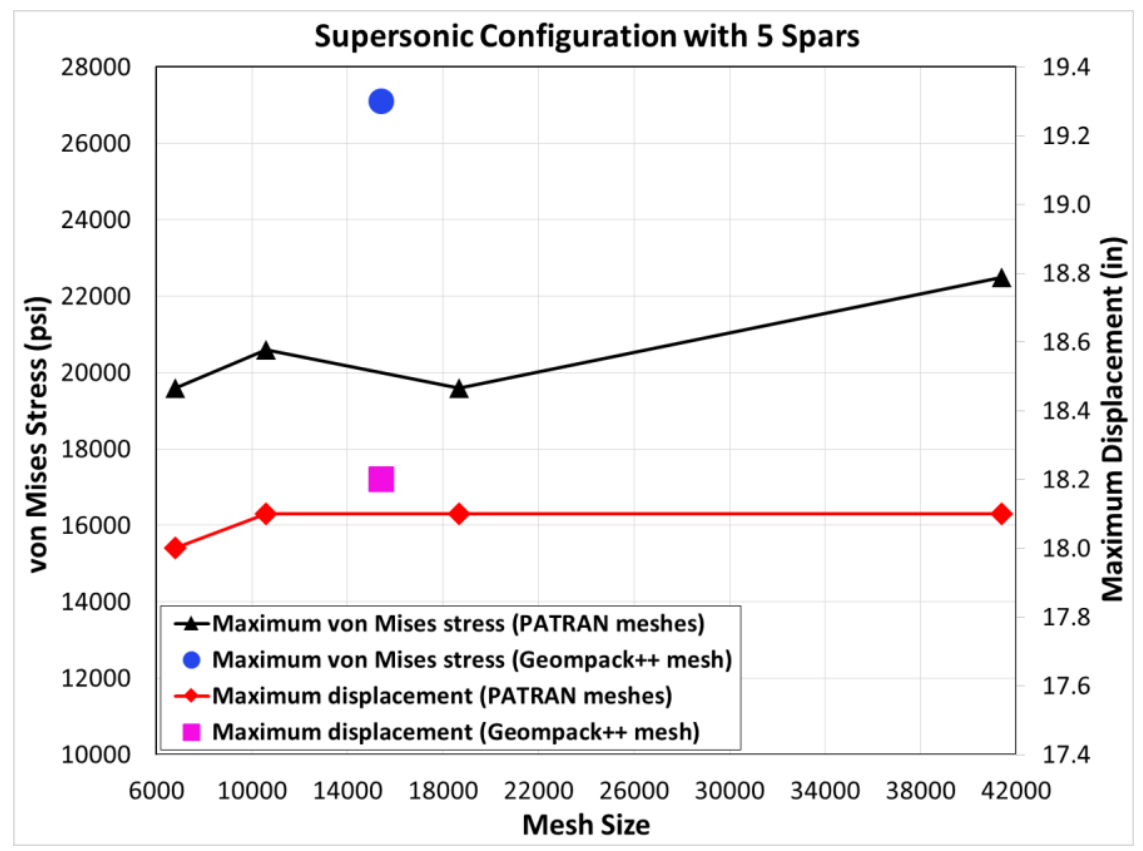

Figure 21. Sensitivity of maximum stress and displacement to mesh size.

\section{Concluding Remarks}

A novel process of using FEM-ready geometry and commercial meshing tools for automated generation of finiteelement meshes is developed. The automation process uses a set of conceptual-level parameters to define internal structural components and generate a fully connected finite-element mesh for any fuselage-wing configuration. A simple thickness optimization problem is used to demonstrate the feasibility of the automation process for finiteelement analysis in conceptual design phase. The automation process is implemented in ModelCenter. It starts with an OpenVSP geometry and ends with a NASTRAN 200 solution for optimal thickness distribution. The set-up time for a given fuselage-wing configuration measures in minutes instead of hours or days.

American Institute of Aeronautics and Astronautics 
Four structural layouts for supersonic and subsonic configurations are used to demonstrate versatility and robustness of the automation process. A total of 20 meshes for the four different structural layouts are generated and hard convergence of NASTRAN 200 solution is achieved for every mesh. Even though 11-16\% differences in the optimal structural weights of a given structural layout are observed for meshes of different sizes (approximately 6,80041,000 elements), the relative difference of the optimal structural weights for two finest meshes is within 5.7\%. Numerical discrepancy up to $4 \%$ in the optimal structural weight is observed due to the choice of initial guess for design variables. All solutions for the same structural layout exhibit similar characteristics in terms of thickness distribution and stress contour pattern. The stress contour plot for a coarse mesh resembles a smeared version of that for a fine mesh. Using the same thickness distribution for the five meshes of the supersonic configuration with 5 spars, the maximum displacements for these meshes differ by at most $1.1 \%$, while noticeable differences between the maximum von Mises stresses are observed.

In future, the automation process will be extended to include other aerodynamic surfaces (such as horizontal and vertical tails), non-structural masses (such as landing gear, fuel, and engines), and aerodynamic load conditions in the structural model for static aeroelastic analysis.

\section{Acknowledgments}

The authors would like to thank Karl Geiselhart at NASA Langley Research Center for providing the OpenVSP model, FLOPS weight data, and reference info of the subsonic business jet G450. The authors would also like to acknowledge James Fenbert at Analytical Mechanics Associates for instrumental comments on the paper. This work is funded by NASA Commercial Supersonic Technology Project and NASA Transformational Tools \& Technologies Project.

\section{References}

${ }^{1}$ Gern, F., "Update on HCDstruct - A Tool for Hybrid Wing Body Conceptual Design and Structural Optimization," AIAA 2015-2544, January 2015.

${ }^{2}$ Laughlin, T., Corman, J., and Mavris D., "A Parametric and Physics-Based Approach to Structural Weight Estimation of the Hybrid Wing Body Aircraft," AIAA 2013-1082, January 2013.

${ }^{3}$ NASTRAN, Version 2014.0, MSC Software, http://www.mscsoftware.com/product/msc-nastran [cited 2 March 2016].

${ }^{4}$ Eldred, L., Padula, S., and Li, W., "Enabling Rapid and Robust Structural Analysis," NASA/TM-2015-218687, 2015.

${ }^{5}$ RapidFEM, M4 Engineering, www.m4-engineering.com/software-creation/rapidfem [cited 2 March 2016].

${ }^{6}$ OpenVSP, Open Source, http://www.openvsp.org [cited 2 March 2016].

${ }^{7}$ Hahn, A., "Vehicle Sketch Pad: A Parametric Geometry Modeler for Conceptual Aircraft Design," AIAA 2010-657, January 2010.

8 Joe, B., "Geompack++ Meshing Operations," Technical Report ZCS2012-01, Sep 2012.

${ }^{9}$ Geompack++, Zhou Computing Services Inc., http://members.shaw.ca/bjoe [cited 2 March 2016].

${ }^{10}$ PATRAN, Version 2014 64-Bit, MSC Software, http://www.mscsoftware.com/product/patran [cited 2 March 2016].

${ }^{11}$ ModelCenter, Version 11.2, Phoenix Integration Inc., www.phoenix-int.com [cited 2 March 2016].

${ }^{12}$ Tecplot 360 EX, Version 2015 R1, Tecplot Inc., http://www.tecplot.com/products/tecplot-360 [cited 2 March 2016].

${ }^{13} \mathrm{Li}$, W., "Feasibility of Supersonic Aircraft Concepts for Low-Boom and Flight Trim Constraints," AIAA 20152581, June 2015.

${ }^{14}$ de Boor, C., "A Practical Guide to Splines (Revised Edition)," Springer, New York, 2001.

${ }^{15}$ Initial Graphics Exchange Specification, IGES 5.3, U.S. Product Data Association, 1996.

${ }^{16}$ Joe, B., "Geompack++ File Formats for Regions and Meshes," Technical Report ZCS2008-01, March 2008.

${ }^{17}$ McCuller, L., "FLOPS User Guide," NASA Langley Research Center, Hampton, Virginia, 2008.

${ }^{18}$ Gulfstream G450 Sketch, http://www.flightglobalimages.com/p/241/gulfstream-g450-cutaway-poster-1571293.jpg [cited 2 March 2016].

American Institute of Aeronautics and Astronautics 